\title{
An antitumorigenic role for the IL-33 receptor, ST2L, in colon cancer
}

\author{
Charlotte O'Donnell ${ }^{1,7}$, Amr Mahmoud ${ }^{2,7}$, Jonathan Keane ${ }^{1,3}$, Carola Murphy ${ }^{4}$, Declan White ${ }^{2,3}$, \\ Sinead Carey ${ }^{5}$, Micheal O'Riordain ${ }^{3,6}$, Michael W Bennett ${ }^{5}$, Elizabeth Brint ${ }^{\star 2,3,8}$ and Aileen Houston ${ }^{1,3,8}$ \\ ${ }^{1}$ Department of Medicine, University College Cork, Cork, Ireland; ${ }^{2}$ Department of Pathology, University College Cork, Cork, \\ Ireland; ${ }^{3}$ Alimentary Pharmabiotic Centre, University College Cork, Cork, Ireland; ${ }^{4}$ Cork Cancer Research Centre, University \\ College Cork, Cork, Ireland; ${ }^{5}$ Department of Histopathology, Cork University Hospital, Cork, Ireland and ${ }^{6}$ Department of Surgery, \\ Mercy University Hospital, Cork, Ireland
}

Background: Despite the importance of inflammation in cancer, the role of the cytokine IL-33, and its receptor ST2, in colon cancer is unclear. The aim of this study was to investigate the role of IL-33, and its receptor isoforms (ST2 and ST2L), in colon cancer.

\begin{abstract}
Methods: Serum levels of IL-33 and sST2 were determined with ELISA. ST2 and IL-33 expression was detected with quantitative real-time PCR (qRT-PCR), western blotting and immunohistochemistry. ST2 expression in CT26 cells was stably suppressed using ST2-specific shRNA. Cytokine and chemokine gene expression was detected with qRT-PCR.

Results: Human colon tumours showed lower expression of ST2L as compared with adjacent non-tumour tissue $(P<0.01)$. Moreover, the higher the tumour grade, the lower the expression of ST2L $(P=0.026)$. Colon cancer cells expressed ST2 and IL-33 in vitro. Functional analyses showed that stimulation of tumour cells with IL-33 induced the expression of chemokine (C-C motif) ligand 2 (CCL2). Knockdown of ST2 in murine colon cancer cells resulted in enhanced tumour growth $(P<0.05)$ in BALB/c mice in vivo. This was associated with a decrease in macrophage infiltration, with IL-33-induced macrophage recruitment reduced by antagonising CCL2 in vitro.
\end{abstract}

Conclusion: The IL-33/ST2 signalling axis may have a protective role in colon carcinogenesis.

Interleukin (IL)-33 is a member of the IL- 1 superfamily, identified in 2005 as the ligand for the receptor ST2. Differential mRNA processing within the ST2 gene generates three isoforms of ST2 proteins: a transmembrane receptor (ST2L), a secreted soluble form (sST2) and a variant form (ST2V; Garlanda et al, 2013). Initial studies on the role of ST2L in the immune system demonstrated that ST2L is expressed on many immune cells, including macrophages, mast cells, innate lymphoid cells and $\mathrm{T}$ cells, in particular Th2 cells (Lu et al, 2015). Moreover, signalling through ST2L promotes the development of Th2 cells, and the induction of the Th2 cytokines IL-4, IL-5 and IL-13. sST2 is thought to act as a decoy receptor, binding to IL-33, whereas the function of ST2V is unclear. More recently, research on IL-33/ST2 has predominantly focused on their role in chronic disorders, with IL-33 and ST2 involved a role in the pathogenesis of several human inflammatory diseases including inflammatory bowel disease (Pastorelli et al, 2010; Sedhom et al, 2013).

Given the importance of inflammation in cancer, recent studies have begun to investigate the role of IL-33 and ST2 in carcinogenesis. Interleukin-33 has been shown to be elevated in the serum of patients with cancer, including that of gastric, lung and hepatocellular cancer patients (Chen et al, 2014), with high levels of sST2 detected in the serum of metastatic breast cancer (Gillibert-Duplantier et al, 2012) and hepatocellular cancer patients (Bergis et al, 2013). Studies in breast cancer have suggested that the IL-33/ST2 pathway inhibits antitumour immunity, subsequently

*Correspondence: Dr E Brint; E-mail: e.brint@ucc.ie

${ }^{7}$ Joint first authors.

${ }^{8}$ These authors contributed equally to this work.

Revised 6 November 2015; accepted 18 November 2015; published online 17 December 2015

(c) 2016 Cancer Research UK. All rights reserved 0007-0920/16 
promoting breast cancer metastasis (Jovanovic et al, 2014). Other studies have implicated a role for IL-33 and ST2 in tumour cell invasion and metastasis, with inhibition of IL-33 in glioma cells and colon cancer cells resulting in reduced tumour growth, migration and colony formation in vitro, and smaller tumours in vivo (Fang et al, 2014; Liu et al, 2014). Interleukin-33 was also shown to activate tumour stroma and promote polyposis in APC (Min/ + ) mice (Maywald et al, 2015). In contrast, a protective role for IL-33 has been reported in other studies, with IL-33 reduced in the plasma of non-small-cell lung cancer relative to controls (Barrera et al, 2015), and IL-33 negatively correlating with the tumour stage in multiple myeloma patients (Musolino et al, 2013). Moreover, whereas expression of both IL-33 and ST2 was shown to be increased in intestinal adenomas, expression of both proteins was reduced in colorectal carcinomas (Cui et al, 2015). Moreover, overexpression of IL-33 in tumours resulted in an increase in the antitumour immune response and tumour rejection through the activation of $\mathrm{CD}^{+}{ }^{+} \mathrm{T}$ cells and natural killer (NK) cells (Gao et al, 2015). Most studies to date, however, have focused predominantly on the role of IL-33 in cancer, with less known about the role of the receptor. Here we demonstrate that suppression of ST2 expression by colon tumour cells results in increased tumour growth in vivo, with progressively lower expression of ST2L by tumour cells observed with increasing tumour stage.

\section{MATERIALS AND METHODS}

Study populations. The study protocol, including all procedures and study populations, was approved by the University College Cork Clinical Research Ethics Committee of the Cork Teaching Hospitals (ECM (3) P 3 September 2013). For patient cohort 1, 24 fresh samples of human colon cancer and paired normal tissues were obtained during surgery at the Mercy University Hospital Cork. A blood sample was obtained from each patient before surgery for serum analysis. All samples were collected with patients' informed consent. For patient cohort 2, 68 paraffinembedded formalin-fixed samples were obtained from the archives of Cork University Hospital. Relevant patient history was also extracted from the archives. Control serum samples were obtained from healthy volunteers aged between 18 and 65 years. Patient demographics are contained in Supplementary Table 1.

Reagents. Recombinant $\mathrm{TNF} \alpha$ was obtained from PeproTech (Rocky Hill, NJ, USA), EGF was purchased from Biolegend Inc (San Diego, CA, USA) and $\mathrm{PGE}_{2}$ was obtained from Cayman Company (Ann Arbor, MI, USA). LPS was purchased from Invivogen (San Diego, CA, USA). Recombinant IL-33 was obtained from ProSpec (East Brunswick, NJ, USA). The primary antibodies used were as follows: polyclonal anti-human anti-ST2L antibody (cat \# NBP1-85251) Novus, (Littleton, CO, USA), anti-mouse antiST2 antibody (cat\#ab25877) Abcam (Cambridge, UK), anti-human anti-IL-33 antibody (Nessy-1) (cat\#ALX-804-840) Enzo Life Sciences (Farmingdale, NY, USA). APC/Cy7 anti-mouse Ly-6G/ Ly-6C antibody and PE anti-mouse F4/80 antibody were obtained from Biolegend Inc. FITC rat anti-mouse CD8 was purchased from BD Biosciences (Franklin Lakes, NJ, USA).

Mice and tumour model. Female BALB/c mice (4-6 weeks) were obtained from Harlan UK (Oxon, UK) and experiments performed in accordance with the institutional guidelines using an Animal Experimentation Ethics Committee-approved protocol (approval 2012/051). To establish subcutaneous tumours, mice were injected into the right flank with $4.0 \times 10^{4}$ CT26 cells resuspended in $200 \mu \mathrm{l}$ phosphate-buffered saline (five mice per group). Tumour growth was monitored by regular measurement of tumour length $(a)$ and width $(b)$ using a Vernier calliper, and the volume was calculated as follows: $1 / 2 \times\left(a \times b^{2}\right)$. Animals were killed 20 or 24 days later and tumours excised. The acceptable maximum tumour volume for mice to be killed was $2 \mathrm{~cm}^{3}$, in accordance with the ethical guidelines.

Generation of ST2 ${ }^{\text {Low/negative }}$ CT26 cells. Cells were transfected with lentiviral particles containing target-specific shRNAs against ST2 (sc-40036-V) or control lentiviral particles containing scrambled shRNA (sc-108080; Santa Cruz Biotechnology, Dallas, TX, USA), according to the manufacturers' instructions. Puromycin-resistant clones were selected by limiting dilution.

Quantitative real-time PCR. Total RNA was isolated using the GenElute Mammalian Total RNA Mini kit (Sigma-Aldrich, Dorset, $\mathrm{UK}$ ) according to the manufacturer's instructions, and cDNA was synthesised. Individual PCR primer pairs (Supplementary Table 2) and probes were designed using the Roche Universal Probe Library Assay Design Centre. The $2^{-\triangle \Delta \mathrm{CT}}$ method was used to calculate relative changes in the gene expression.

Immunohistochemistry. Immunohistochemical staining was performed on formalin-fixed, paraffin-embedded (FFPE) sections as previously described (Fernandes et al, 2015). A semiquantitative scoring system was developed, with each tumour given a score according to the intensity of staining.

Proliferation assay. Cell proliferation was determined by analysing BrdU incorporation into newly synthesised DNA using a Cell Proliferation ELISA System (Roche Molecular Biochemicals, Mannheim, Germany) according to the manufacturer's instructions.

Flow cytometry. Single-cell suspensions were prepared by collagenase/dispase digestion. Overall, 200000 cells were re-suspended in $200 \mu \mathrm{l}$ of cell-staining buffer, incubated with antibodies to $\mathrm{LY} 6 \mathrm{G}, \mathrm{CD} 8$ or $\mathrm{F} 4 / 80$ for $30 \mathrm{~min}$ at $4{ }^{\circ} \mathrm{C}$ in the dark, and then fixed in $1 \%$ paraformaldehyde. Relative fluorescence intensities were measured using a FACSLSRII 5 laser (UV/violet/blue/ yellowgreen/red) cytometer and BD Diva software (Becton Dickinson, Oxford, UK). For each sample, 20000 events were recorded. Dead cells were gated out of the analysis by staining with the viability dye VIVID (Invitrogen). The percentage of cells labelled with each antibody was calculated in comparison with cells stained with isotype control antibody. Analysis gates for each antibody were set by using fluorescence minus one controls with a threshold below 1\% (Supplementary Figure 3).

Statistical analysis. Experiments were performed a minimum of three times in triplicate. Results were statistically evaluated using one-way ANOVA with Dunnetts post test, or by Student's paired $t$-test. The Kendall tau-b correlation coefficient was used to evaluate the association between ST2L expression and tumour stage. Kaplan-Meier analysis was used to calculate survival rates, and differences in survival curves were determined by the log-rank test.

\section{RESULTS}

Expression of ST2L in CRC is lower compared with adjacent non-tumour tissue, with expression being progressively lower with higher tumour grade. In order to examine potential alterations in expression of both IL-33 and its receptor ST2 in colorectal cancer stage (CRC), a cohort of 24 patients with CRC was recruited, 8 of whom had received chemotherapy before surgery. Initially, expression levels of IL-33 and ST2 were examined using quantitative real-time PCR (qRT-PCR). There was no change in the level of transcription of either IL-33 or total ST2 between CRC and adjacent non-tumour tissue (Figure 1A). We subsequently designed and utilised a primer specific to the 

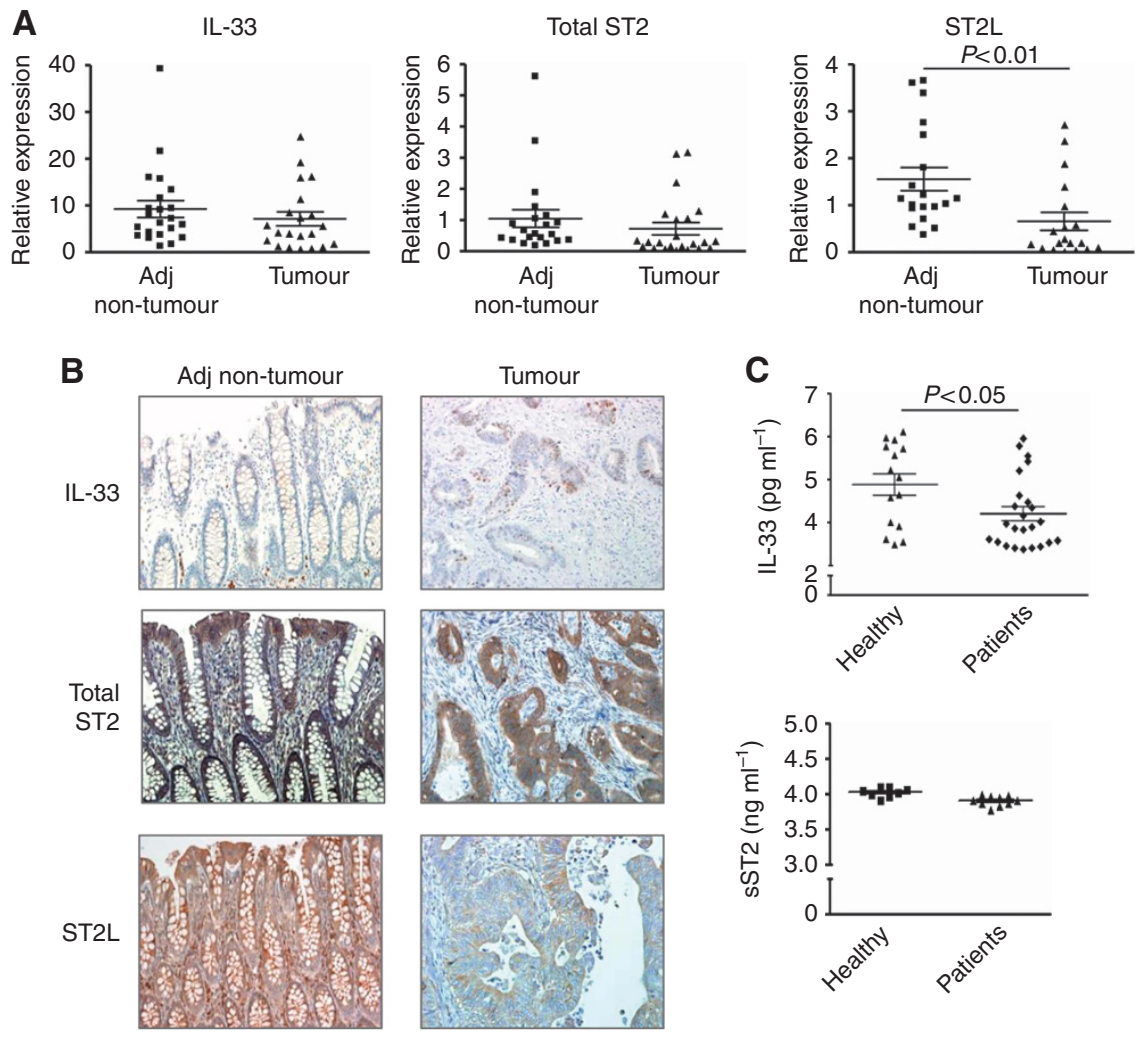

Figure 1. ST2L expression is lower in colorectal cancer tissue relative to adjacent non-tumour tissue. (A) RNA was extracted and changes in IL-33, total ST2 and ST2L detected using qRT-PCR. (B) Immunohistochemical staining for IL-33, ST2 and ST2L was performed on paraffin-embedded tissue sections. Original magnification: $\times 20$. Images shown are representative of the findings obtained. (C) Serum was obtained from healthy individuals and patients with CRC, and IL-33 and SST2 levels determined using ELISA.

intracellular portion of ST2 to examine changes in expression of ST2L in colon cancer. The level of ST2L mRNA was significantly lower $(P=0.0067)$ in CRC tissue as compared with adjacent nontumour tissue (Figure 1A). Alterations in expression were confirmed at the protein level using immunohistochemistry (IHC). No change was observed in either IL-33 or total ST2, although staining intensity for ST2L was consistently lower in CRC as compared to adjacent non-tumour tissue. Representative images are shown in Figure 1B. Serum levels of IL-33 and sST2 were also examined in CRC patients $(n=24)$ compared with healthy volunteers $(n=15)$, with a slight decrease in IL-33 $(P=0.0252)$ and no alterations of sST2 (Figure 1C) observed.

We subsequently wished to identify whether there was any association between expression of ST2L and tumour grade. To investigate this, IHC for ST2L was performed on 68 FFPE tissue sections of various stages of CRC, 17 of whom had received chemotherapy. A scoring system was developed, with the staining intensity of ST2L categorised as negative, weak, moderate or strong. As the tumour stage increased, the intensity of ST2L expression observed was lower, with expression of ST2L negatively correlating with tumour grade (Kendall tau-b: $-0.248, P=0.026$ ). Fifty-seven per cent of stage I tumours showed moderate to strong expression, with only $15 \%$ of stage IV tumours showing moderate/ strong expression (Table 1). Representative images of the ST2L staining obtained for each stage are shown in Figure 2A.

The relationship between the expression levels of ST2L and clinical prognosis in cohort 2 was also assessed. Clinicopathological correlation for these patients was evaluated by determining the patients' survival and clinical progression. There was a trend towards improved overall survival when tumours had strong to moderate expression of ST2L, although this was not statistically significant $(P=0.0838$; Figure $2 \mathrm{~B})$.

\section{Table 1. ST2L expression in colon tumour cells}

\begin{tabular}{|l|c|c|c|}
\hline CRC stage & Total & \% Negative/weak & \% Moderate/strong \\
\hline Stage I & 14 & 43 & 57 \\
\hline Stage II & 21 & 62 & 38 \\
\hline Stage III & 20 & 65 & 35 \\
\hline Stage IV & 13 & 85 & 15 \\
\hline $\begin{array}{l}\text { Abbreviation: CRC= colorectal cancer stage. Expression of ST2L decreases with increasing } \\
\text { tumour stage. Tumour sections were scored as negative, weak, moderate or strong, } \\
\text { according to the intensity of ST2L staining. Kendall tau-b: }-0.248, P=0.026 .\end{array}$ \\
\hline
\end{tabular}

No association was observed between ST2L expression and chemotherapy treatment. Approximately half of the samples derived from patients receiving chemotherapy were negative for ST2L (9 out of 17) and (5 out of 8), with the remainder all expressing ST2L of varying intensities.

Colon cancer cell lines express ST2L and IL-33, with IL-33 stimulation not increasing cell proliferation or invasion, but inducing migration in vitro. To further characterise and examine the role of ST2 and IL-33 in colon cancer, expression of both ST2L and IL-33 was examined in several colon cancer cell lines. Basal expression of ST2L was low in all three cell lines examined, with expression increased upon stimulation with the pro-inflammatory stimuli, LPS, TNF $\alpha$ and $\mathrm{PGE}_{2}$ (Figure $3 \mathrm{~A}$ ). Interleukin-33 protein was also detected in all cell lines, with no change in expression seen upon stimulation with any of the pro-inflammatory cytokines investigated (Figure 3A). In contrast, IL-33 was expressed at a very low basal level in both B16F10 melanoma and A549 lung cancer cells, with expression induced upon stimulation with LPS. Moreover, these cells had high basal levels of ST2L, which was unaltered by either LPS or TNF $\alpha$ (Supplementary Figure 1). 
$\mathbf{A}_{(\mathrm{i})}$

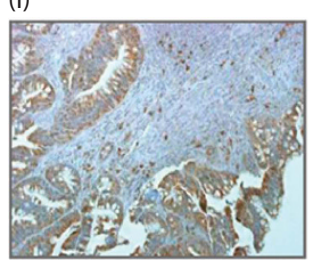

(ii)

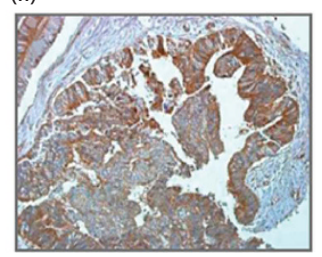

(iii)

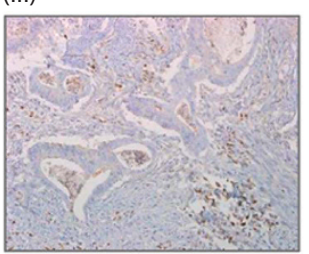

(iv)

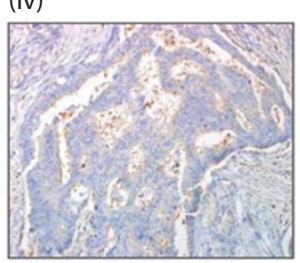

B

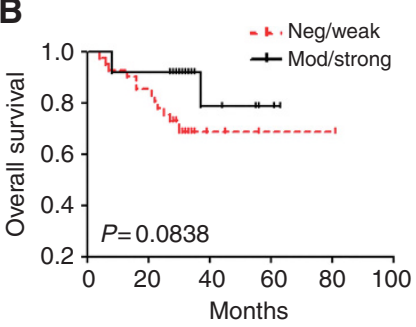

Figure 2. ST2L expression is progressively lower with increasing tumour stage. (A) Immunohistochemical staining for ST2L was performed on paraffin-embedded colonic tissue sections. Original magnification: $\times 20$. Images shown are representative of the findings obtained for each stage. (B) The relationship between ST2L expression in CRC cells and clinical prognosis in patients with CRC was analysed by Kaplan-Meier analysis.

Differences in survival curves were determined by the log-rank test.

A

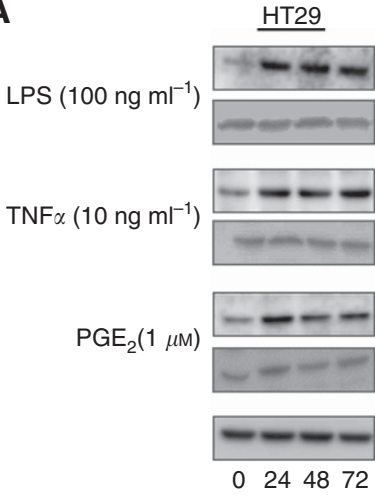

SW480
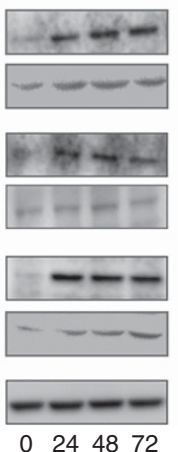

0244872

SW480
$\underline{\mathrm{CT} 26}$

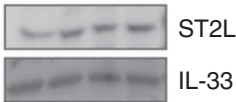

IL-33
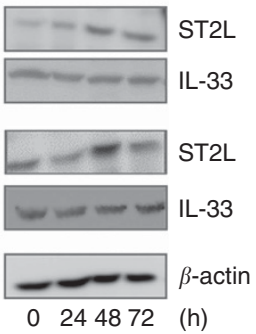

CT26

B

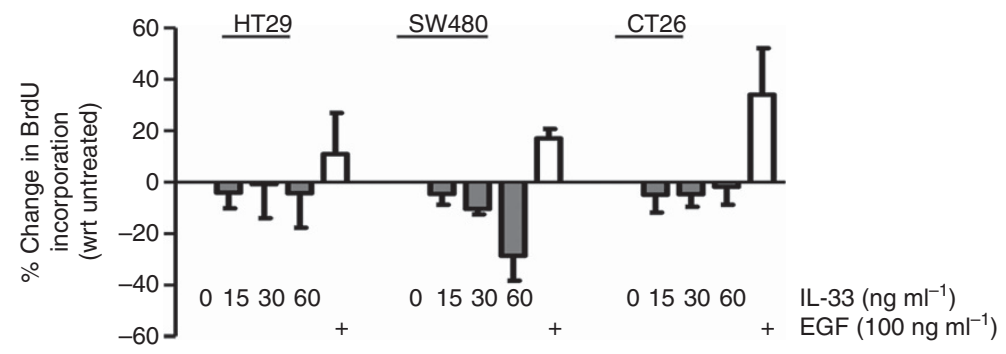

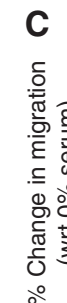

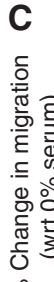

IL-33 (ng
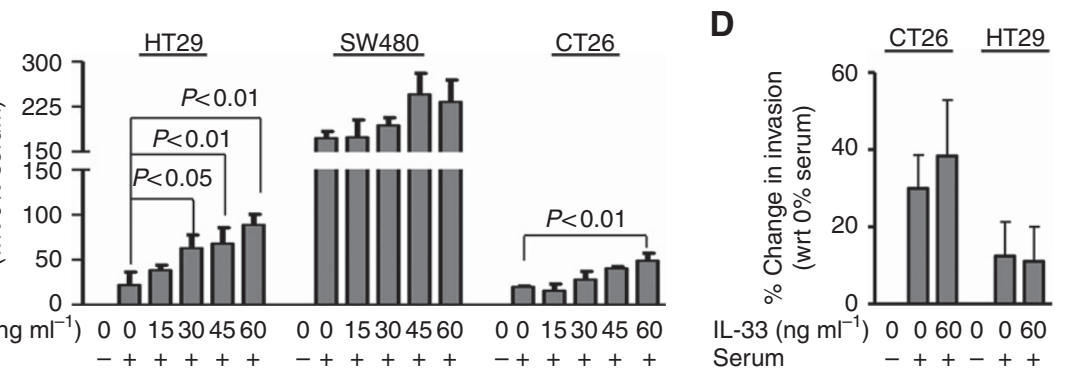

Figure 3. Pro-inflammatory cytokines induce the expression of ST2L but not IL-33 in colon cancer cells, with IL-33 inducing migration but not proliferation or invasion in vitro. (A) Cells were stimulated for $24 \mathrm{~h}$ with LPS, TNF $\alpha$ and PGE 2 as indicated, and changes in ST2L and IL-33 detected. Immunoblotting for $\beta$-actin was used as the loading control. Data shown are representative of three independent experiments. Cells were stimulated with IL-33 as indicated and changes in (B) proliferation assessed by BrdU incorporation, with EGF used as a positive control, (C) migration assessed using a modified Boyden chamber assay and (D) invasion assessed using a Matrigel invasion assay system. Data shown are mean \pm s.e.m. $(n=3)$.

Colon cancer cells were stimulated with increasing concentrations of IL-33 and changes in proliferation and migration examined. Interleukin-33 treatment resulted in a slight decrease in cellular proliferation (Figure 3B). In contrast, IL-33 increased the migration of the cells over the levels observed towards $10 \%$ serum. This increase in migration occurred in a dose-dependent manner for all cell lines (Figure 3C). Interleukin-33, however, had no effect on cell invasion (Figure 3D).
Interleukin-33 induces limited expression of cytokines/ chemokines in colon cancer cells. As we had observed expression of ST2L in unstimulated cells, we next investigated whether IL-33 induced the transcription of cytokines, chemokines or angiogenic factors. Stimulation of HT29 (Figure 4A) and SW480 (Figure 4B) human colon cancer cells with IL-33 had no effect on transcription of a panel of factors, with the exception of CCL2, which was strongly induced in both cell lines. In contrast to the human cell 
lines, stimulation of murine CT26 cells with IL-33 induced an increase in VEGF, CXCL-1 and CCL2 (Figure 4C).

Suppression of ST2 expression in colon cancer cells results in increased tumour growth in vivo. We next investigated the biological effect of reducing ST2 expression in colon cancer cells in a murine model. ST2 expression was reduced in CT26 mouse colon cancer cells using shRNA, the suppression of ST2 being confirmed using western blot analysis (Figure 5A). Suppression of ST2 did not affect either the basal level of proliferation (Figure 5B), proliferation induced by pro-inflammatory stimuli (Supplementary Figure 2A) or migration (Figure 5C) of these cells. Consistent

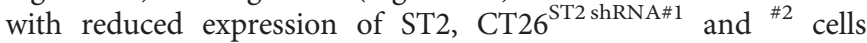

showed markedly reduced transcription of CXCL-1 following IL-33 stimulation (Figure 5D). Immunocompetent $\mathrm{BALB} / \mathrm{c}$ mice were injected subcutaneously with CT2 $6^{\text {scr shRNA\#1 }}$, CT2 $6^{\text {ST2 shRNA\#1 }}$ or CT26 ${ }^{\text {ST2 }}$ shRNA\#2 cells, and tumour growth monitored. All mice developed palpable tumours. However, in contrast to the in vitro findings, whereby suppression of ST2 did not alter cellular proliferation, tumour growth was increased when ST2 was suppressed in advance by shRNA (Figure 5E; $P<0.05$ ).

These findings were confirmed in a second independent study using additional CT2 $6^{\text {scr shRNA\#2 }}$ and CT26 $6^{\text {ST2 shRNA\#3 }}$ clones that were subsequently derived. In this second study, the increase in tumour growth following suppression of ST2 was even more pronounced $(P=0.0045$; Supplementary Figure $2 \mathrm{~B}-\mathrm{D})$.
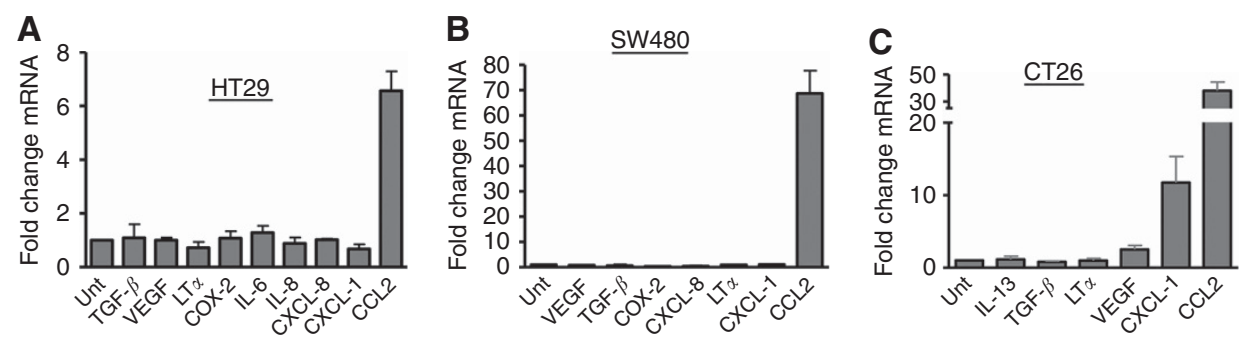

Figure 4. IL-33 induces the expression of a limited number of cytokines/chemokines in colon cancer cells. (A-C) Cells were stimulated with $15 \mathrm{ng} \mathrm{ml}^{-1}$ of IL-33. RNA was extracted $4 \mathrm{~h}$ later, and changes in cytokine/chemokine expression detected using qRT-PCR. Data shown are mean \pm s.e.m. $(n=3)$.
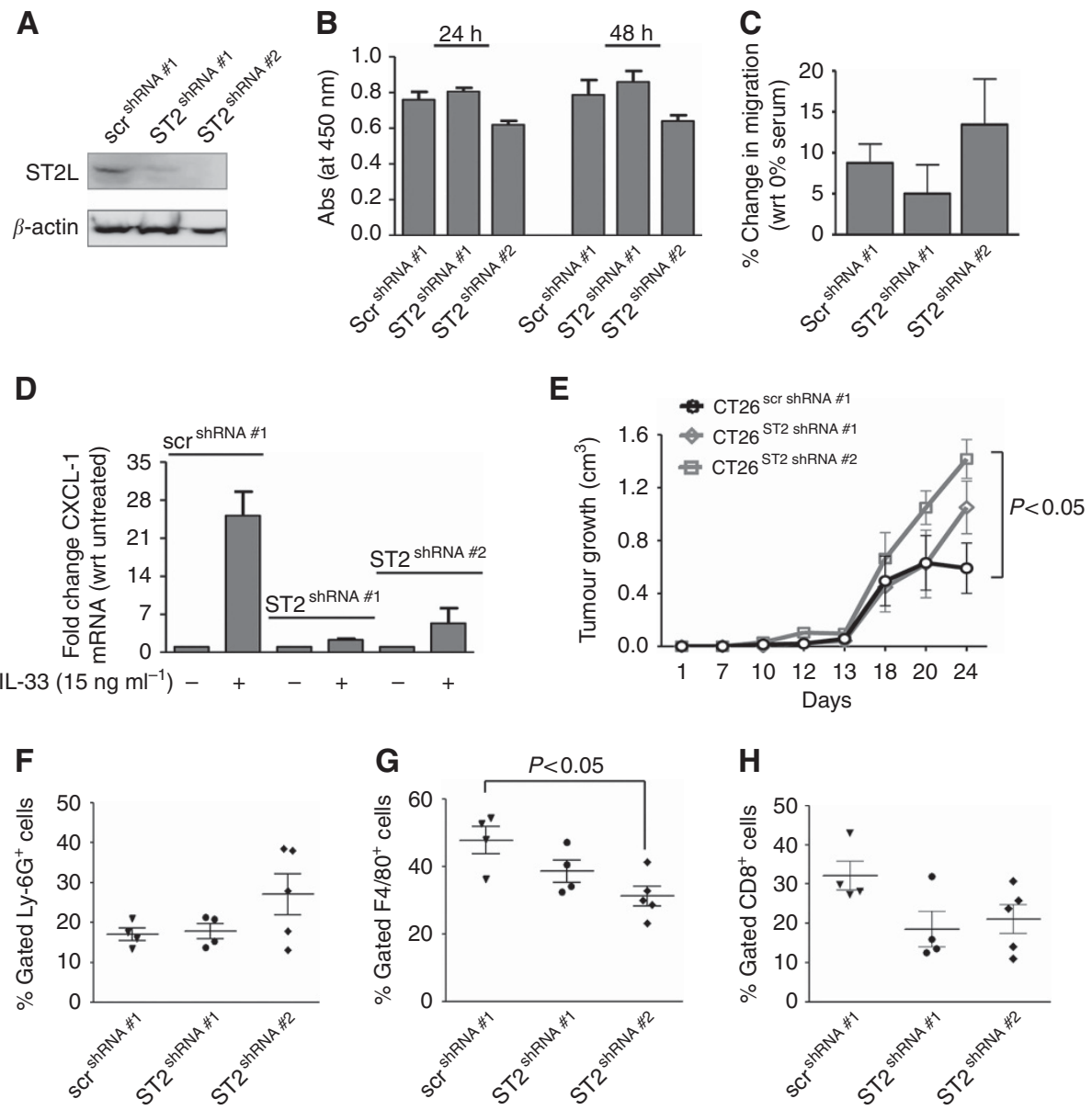

Figure 5. Suppression of ST2 expression by colon cancer cells results in increased tumour cell growth in vivo. (A, D) ST2 expression was

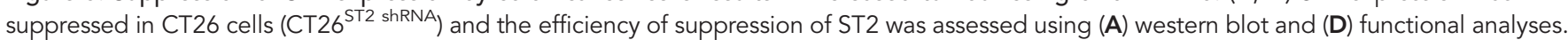
Changes in basal levels of (B) proliferation and (C) migration were assessed. (E) Cells were subcutaneously injected into BALB/c mice and tumour growth monitored. (F-H) Tumours were excised and infiltration of $\mathrm{Ly}_{-} 6 \mathrm{G}^{+}, \mathrm{F} 4 / 80^{+}$and $\mathrm{CD} 8^{+}$cells assessed using flow cytometry. 
Tumours were excised and immune cells analysed using flow cytometry. Although suppression of ST2 in tumours did not affect neutrophil infiltration (Figure 5F), there was a reduction in macrophage recruitment following ST2 knockdown in the tumour cells (Figure 5G). Although not significant, there was also a decrease in the recruitment of $\mathrm{CD}^{+} \mathrm{T}$ cells (Figure 5H). Representative dot plots for the flow cytometric analysis are shown in Supplementary Figure 3.

IL-33 induces the recruitment of macrophages in vitro, with

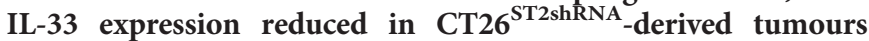
in vivo. It is well established that, if appropriately activated, macrophages can mediate tumour rejection (Edin et al, 2013). Given the reduction seen in macrophage infiltration in tumours derived from CT26 $6^{\mathrm{ST} 2}$ shRNA\#1 and ${ }^{\# 2}$ cells, we investigated whether there was an increase in macrophage migration towards supernatant from IL-33-stimulated tumour cells, relative to untreated tumour cell supernatant. There was an $\sim 25 \%$ increase in migration of macrophages as compared with unstimulated CT26 cell supernatant (Figure 6A). Consistent with the induction of CCL2 by IL-33, a blocking antibody to CCL2 reduced macrophage migration towards the supernatant of IL-33-stimulated CT26 cells $(P<0.05$; Figure 6A).

We finally examined expression of IL-33 within the murine tumours. A marked reduction was observed in IL-33 expression within the CT26 $6^{\text {ST2 }}$ shRNA tumours as compared with CT26 $6^{\text {ScrshRNA }}$ tumours. Although the underlying mechanism for this reduction in IL-33 in vivo is unclear, it may be due to a lack of positive regulation of IL-33 by ST2L signalling. In conclusion, these data suggest that the alterations in tumour growth in vivo following suppression of ST2 is likely due, at least in part, to a reduction in tumour-expressed IL-33, perhaps due to a reduction in tumourderived macrophage chemotactic factors, and consequent reduction in macrophage recruitment.

\section{DISCUSSION}

In this study we have demonstrated, using both a preclinical in vivo model of tumour development involving ectopically injected colon carcinoma cells, as well as human clinical samples, that signalling through ST2 on tumour cells likely has an antitumorigenic role in CRC. We have shown that suppressing ST2 expression in colon tumour cell lines increased tumour growth in vivo. Expression of ST2L was found to be lower in human tumours relative to adjacent non-tumour tissue, with the higher the tumour grade, the lower the expression of ST2L.

Several recent reports have demonstrated an antitumorigenic role for the IL-33-ST2 axis. In these studies, IL-33 was shown to promote the function of $\mathrm{CD}^{+} \mathrm{T}$ cells and NK cells, thereby promoting tumour eradication (Yang et al, 2011). Consistent with this, studies examining T-cell infiltration in CRC have shown that high levels of $\mathrm{CD} 8^{+} \mathrm{T}$-cell infiltration is associated with improved overall patient survival (Naito et al, 1998). Macrophages can also act as potent antitumour immune effector cells (Edin et al, 2013). In our study, lack of ST2 expression in tumours was associated with a reduction in both macrophage and $\mathrm{CD}^{+} \mathrm{T}$ cell, and augmented tumour growth. Consistent with a potential antitumorigenic role for ST2, we have shown that a low level of ST2 is associated with poor prognosis and overall survival in human CRC. Thus, our data indicate that the reduction in ST2 expression by tumour cells may result in reduced signalling through ST2, affecting the tumour microenvironment by altering the cell composition of the microenvironment, favouring increased tumour growth.

Recent studies investigating the role of IL-33/ST2 in colon cancer have shown somewhat divergent effects (Liu et al, 2014; Cui et al, 2015; Maywald et al, 2015). In contrast to our findings, these studies reported an increase in IL-33 in CRC as compared with adjacent normal tissue and healthy volunteers, with IL-33 having a protective antitumorigenic effect in CRC (Liu et al, 2014). Interestingly, the level of IL-33 varied greatly between the studies. Similar to our findings, the level of IL-33 seen in CRC was low in two studies (Liu et al, 2014; Cui et al, 2015), with only Maywald et al (2015) reporting high levels of IL-33 in tumours. However, all patients in this study were a heavily pre-treated cohort, receiving 5-fluorouracil-based chemotherapy before analysis. As IL-33 is well known to be released upon cellular damage, it is possible that the chemotherapeutic regimes directly affected the levels of IL-33 in these patients. Indeed, the low level of IL-33 seen in our study may be reflective of the lack of prior chemotherapy in our cohorts. Total ST2 was also reported to be increased in CRC cells relative to adjacent normal cells, in contrast to our findings (Liu et al, 2014). These differences could be because of the isoforms of ST2 examined, given that our observed decrease in expression of ST2 was confined to the transmembrane-bound signalling active L-isoform (ST2L). Indeed, to the best of our knowledge, no other study has specifically examined the expression of ST2L in CRC.

Here we have shown that ST2L expression is upregulated by pro-inflammatory stimuli that have been implicated in colon cancer (LPS, TNF $\alpha$ and $\mathrm{PGE}_{2}$ ), whereas IL-33 appears to be more tightly regulated. This lack of upregulation of IL-33 may well reflect the overall low levels of IL-33 seen in CRC tumour cells. Further characterisation of the function of IL-33 in colon cancer cells revealed that IL-33 did not increase cell proliferation or invasion in vitro, but did induce cell migration. This ability of IL-33 to induce cell migration in vitro suggests that IL-33 may be involved in metastasis in vivo, as suggested by Liu et al (2014). However, we only observed robust migration of colon cancer cells
A

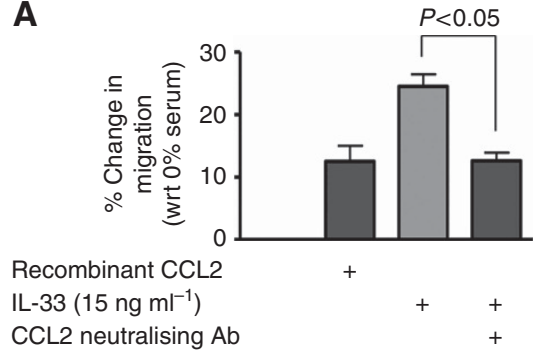

B

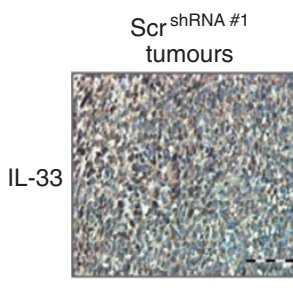

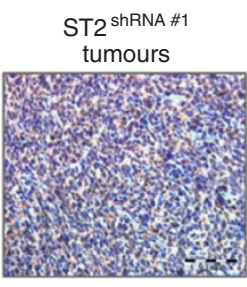
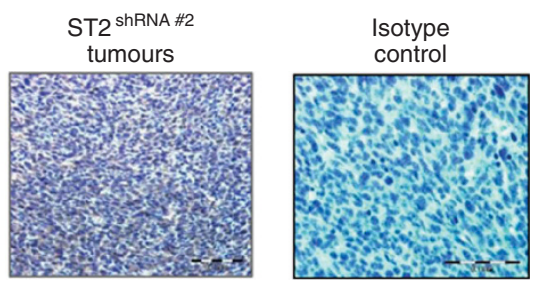

Figure 6. Supernatant from colon cancer cells stimulated with IL-33 show enhanced macrophage migration relative to unstimulated supernatant. (A) Supernatant was isolated from unstimulated or IL-33-stimulated CT26 cells. Neutralising CCL2 antibody was added to the supernatant as indicated. Migration of RAW264.7 macrophages towards the cell culture supernatant was assessed with modified Boyden chamber assay. Data shown are mean \pm s.e.m. $(n=3)$. (B) Immunohistochemical staining for IL-33 was performed on paraffin-embedded murine tumour sections. Original magnification: $\times 40$. Images shown are representative of the findings obtained. 
at higher doses of IL-33, which may not be physiologically relevant. Indeed, IL-33 in the serum of cancer patients, as seen both in this study, and others, was typically in the low $\mathrm{pg} \mathrm{ml}^{-1}$ concentration (Sun et al, 2011; Zhang et al, 2012).

We also comprehensively characterised induction of cytokine and chemokines by IL-33. Interleukin-33 only induced the expression of CCL2 in human cells, with VEGF and CXCL-1 also being induced in murine cancer cells. Other studies investigating induction of cytokines and chemokines in non-haematopoietic cells have also shown a similar lack of induction of cytokines/ chemokines by IL-33 (Masamune et al, 2010). This implies that the response of non-haematopoietic cells to IL-33 stimulation is highly restricted. Indeed, this lack of upregulation of inflammatory cytokines and chemokines is perhaps unsurprising, given the role of IL-33 in maintaining barrier function and homeostasis in the gastrointestinal tract (Lopetuso et al, 2012).

In conclusion, our study has shown a potential antitumorigenic role for ST2L in colon cancer. This is likely mediated by alterations in immune infiltrating cells, in particular through alterations in infiltration by macrophages and $\mathrm{CD}^{+} \mathrm{T}$ cells. Therefore, targeting ST2L may have potential therapeutic benefit for the treatment of colon cancer. However, given that IL-33 and ST2 appear to have differing and opposing functions, depending on the cell type, tissue and organ examined, further work on the role of IL-33 and ST2 in cancer is required.

\section{ACKNOWLEDGEMENTS}

This work was supported by funding from the Health Research Board of Ireland (PhD/2007/4) and the Pathological Society of Great Britain and Ireland (SGS 2014/10/04).

\section{CONFLICT OF INTEREST}

The authors declare no conflict of interest.

\section{REFERENCES}

Barrera L, Montes-Servin E, Barrera A, Ramirez-Tirado LA, Salinas-Parra F, Banales-Mendez JL, Sandoval-Rios M, Arrieta O (2015) Cytokine profile determined by data-mining analysis set into clusters of non-small-cell lung cancer patients according to prognosis. Ann Oncol 26(2): 428-435.

Bergis D, Kassis V, Ranglack A, Koeberle V, Piiper A, Kronenberger B, Zeuzem S, Waidmann O, Radeke HH (2013) High serum levels of the interleukin-33 receptor soluble ST2 as a negative prognostic factor in hepatocellular carcinoma. Transl Oncol 6(3): 311-318.

Chen XJ, Huang YD, Li N, Chen M, Liu F, Pu D, Zhou TY (2014) Correlations between serum IL33 and tumor development: a meta-analysis. Asian Pac J Cancer Prev 15(8): 3503-3505.

Cui G, Qi H, Gundersen MD, Yang H, Christiansen I, Sorbye SW, Goll R, Florholmen J (2015) Dynamics of the IL-33/ST2 network in the progression of human colorectal adenoma to sporadic colorectal cancer. Cancer Immunol Immunother 64(2): 181-190.

Edin S, Wikberg ML, Oldenborg PA, Palmqvist R (2013) Macrophages: good guys in colorectal cancer. Oncoimmunology 2(2): e23038.

Fang KM, Yang CS, Lin TC, Chan TC, Tzeng SF (2014) Induced interleukin-33 expression enhances the tumorigenic activity of rat glioma cells. Neuro Oncol 16(4): 552-566.

Fernandes P, MacSharry J, Darby T, Fanning A, Shanahan F, Houston A, Brint E (2015) Differential expression of key regulators of Toll-like Receptors in Ulcerative Colitis and Crohn's disease: A role for Tollip and
PPARgamma? Clin Exp Immunol; e-pub ahead of print 14 October 2015; doi:10.1111/cei.12732.

Gao X, Wang X, Yang Q, Zhao X, Wen W, Li G, Lu J, Qin W, Qi Y, Xie F, Jiang J, Wu C, Zhang X, Chen X, Turnquist H, Zhu Y, Lu B (2015) Tumoral expression of IL-33 inhibits tumor growth and modifies the tumor microenvironment through CD8 $+\mathrm{T}$ and NK cells. J Immunol 194(1): 438-445.

Garlanda C, Dinarello CA, Mantovani A (2013) The interleukin-1 family: back to the future. Immunity 39(6): 1003-1018.

Gillibert-Duplantier J, Duthey B, Sisirak V, Salaun D, Gargi T, Tredan O, Finetti P, Bertucci F, Birnbaum D, Bendriss-Vermare N, Badache A (2012) Gene expression profiling identifies sST2 as an effector of ErbB2-driven breast carcinoma cell motility, associated with metastasis. Oncogene 31(30): 3516-3524.

Jovanovic IP, Pejnovic NN, Radosavljevic GD, Pantic JM, Milovanovic MZ, Arsenijevic NN, Lukic ML (2014) Interleukin-33/ST2 axis promotes breast cancer growth and metastases by facilitating intratumoral accumulation of immunosuppressive and innate lymphoid cells. Int J Cancer 134(7): 1669-1682.

Liu X, Zhu L, Lu X, Bian H, Wu X, Yang W, Qin Q (2014) IL-33/ST2 pathway contributes to metastasis of human colorectal cancer. Biochem Biophys Res Commun 453(3): 486-492.

Lopetuso LR, Scaldaferri F, Pizarro TT (2012) Emerging role of the interleukin (IL)-33/ST2 axis in gut mucosal wound healing and fibrosis. Fibrogenesis Tissue Repair 5(1): 18.

Lu J, Kang J, Zhang C, Zhang X (2015) The role of IL-33/ST2L signals in the immune cells. Immunol Lett 164(1): 11-17.

Masamune A, Watanabe T, Kikuta K, Satoh K, Kanno A, Shimosegawa T (2010) Nuclear expression of interleukin-33 in pancreatic stellate cells. Am J Physiol Gastrointest Liver Physiol 299(4): G821-G832.

Maywald RL, Doerner SK, Pastorelli L, De Salvo C, Benton SM, Dawson EP, Lanza DG, Berger NA, Markowitz SD, Lenz HJ, Nadeau JH, Pizarro TT, Heaney JD (2015) IL-33 activates tumor stroma to promote intestinal polyposis. Proc Natl Acad Sci USA 112(19): E2487-E2496.

Musolino C, Allegra A, Profita M, Alonci A, Saitta S, Russo S, Bonanno A, Innao V, Gangemi S (2013) Reduced IL-33 plasma levels in multiple myeloma patients are associated with more advanced stage of disease. Br J Haematol 160(5): 709-710.

Naito Y, Saito K, Shiiba K, Ohuchi A, Saigenji K, Nagura H, Ohtani H (1998) $\mathrm{CD} 8+\mathrm{T}$ cells infiltrated within cancer cell nests as a prognostic factor in human colorectal cancer. Cancer Res 58(16): 3491-3494.

Pastorelli L, Garg RR, Hoang SB, Spina L, Mattioli B, Scarpa M, Fiocchi C, Vecchi M, Pizarro TT (2010) Epithelial-derived IL-33 and its receptor ST2 are dysregulated in ulcerative colitis and in experimental Th1/Th2 driven enteritis. Proc Natl Acad Sci USA 107(17): 8017-8022.

Sedhom MA, Pichery M, Murdoch JR, Foligne B, Ortega N, Normand S, Mertz K, Sanmugalingam D, Brault L, Grandjean T, Lefrancais E, Fallon PG, Quesniaux V, Peyrin-Biroulet L, Cathomas G, Junt T, Chamaillard M, Girard JP, Ryffel B (2013) Neutralisation of the interleukin-33/ST2 pathway ameliorates experimental colitis through enhancement of mucosal healing in mice. Gut 62(12): 1714-1723.

Sun P, Ben Q, Tu S, Dong W, Qi X, Wu Y (2011) Serum interleukin-33 levels in patients with gastric cancer. Dig Dis Sci 56(12): 3596-3601.

Yang Q, Li G, Zhu Y, Liu L, Chen E, Turnquist H, Zhang X, Finn OJ, Chen X, Lu B (2011) IL-33 synergizes with TCR and IL-12 signaling to promote the effector function of CD8 + T cells. Eur J Immunol 41(11): 3351-3360.

Zhang P, Liu XK, Chu Z, Ye JC, Li KL, Zhuang WL, Yang DJ, Jiang YF (2012) Detection of interleukin-33 in serum and carcinoma tissue from patients with hepatocellular carcinoma and its clinical implications. J Int Med Res 40(5): 1654-1661.

This work is published under the standard license to publish agreement. After 12 months the work will become freely available and the license terms will switch to a Creative Commons AttributionNonCommercial-Share Alike 4.0 Unported License. 